\title{
Intracellular calcium signaling and phospho-antigen measurements reveal functional proximal TCR activation in lymphocytes from septic shock patients
}

\author{
Charles de Roquetaillade ${ }^{1,2+}$, Khalil Kandara ${ }^{1,3+}$, Morgane Gossez ${ }^{1,3}$, Estelle Peronnet ${ }^{1,4}$, Céline Monard ${ }^{5}$, \\ Martin Cour ${ }^{6}$, Thomas Rimmelé ${ }^{1,5}$, Laurent Argaud ${ }^{6}$, Guillaume Monneret ${ }^{1,3^{*}}$ (i) and Fabienne Venet ${ }^{1,3}$
}

\author{
* Correspondence: guillaume. \\ monneret@chu-lyon.fr \\ ${ }^{+}$Charles de Roquetaillade and Khalil \\ Kandara contributed equally to the \\ work. \\ ${ }^{1}$ EA 7426, Pathophysiology of \\ Injury-Induced Immunosuppression \\ (Université Claude Bernard Lyon 1 - \\ Hospices Civils de Lyon - \\ bioMérieux), Edouard Herriot \\ Hospital, 69437 Lyon, France \\ ${ }^{3}$ Immunology Laboratory, Hôpital E. \\ Herriot - Hospices Civils de Lyon, \\ 69437 Lyon, France \\ Full list of author information is \\ available at the end of the article
}

To the editor,

Sepsis deeply perturbs immune homeostasis by inducing a complex immune response that varies over time and associates a tremendous systemic inflammatory response to anti-inflammatory mechanisms. As a delayed consequence, some septic patients enter a state of profound immunosuppression [1]. As the latter may persist for weeks, leaving the patient at increased risk of secondary infections, immunostimulation recently appeared as a reasonable therapeutic option in patients with signs of persistent and severe immunosuppression [2].

Septic patients develop marked $\mathrm{T}$ lymphocyte dysfunctions such as profound lymphopenia, increased expression of inhibitory co-receptor molecules, decreased repertoire diversity, and reduced functionality (proliferation and cytokine production). These alterations have been repeatedly associated with deleterious outcomes $[1,2]$. However, mechanisms leading to these alterations are only partially understood. For instance, while the role of deactivated mTORC1 is established [3], the intrinsic capacity of $\mathrm{T}$ cell receptor (TCR) to be activated and to transduce intracellular signalling remains unexplored. Among determinants of $\mathrm{T}$ cell response, immediate calcium signaling following TCR ligation is of paramount importance and serves crucial effector functions. Thus, we developed a flow cytometry protocol to follow calcium flux after TCR stimulation in patients' CD4+ T lymphocytes. In addition, phosphorylation of molecules from the proximal and downstream TCR signaling cascade was analyzed (Additional file 1). We included patients with septic shock (according to SEPSIS-3 definition) presenting with features of 
immunosuppression, i.e., decreased monocyte HLA-DR and lymphocyte count (clinical and immunological characteristics in Additional file 1: Table S1).

We show that immediate signaling downstream TCR stimulation was not altered in circulating CD4 lymphocytes from septic shock patients. Indeed, cells exhibited no deregulation of intracytoplasmic calcium influx after TCR ligation compared with healthy controls (OKT3 response, Fig. 1). In agreement, we observed a significant $\mathrm{CD} 3 \zeta$ phosphorylation (one of the first molecules to be phosphorylated after TCR engagement) after $\mathrm{T}$ cell stimulation in both cells from septic patients and controls (Fig. 2). This showed that immediate response after TCR activation was unaffected after sepsis.

In contrast, activation of more distal molecules in the TCR signaling cascade was impacted. For example, stimulation-induced rise of pAkt and pERK was affected leading to a limited mTORC1 activation capacity as measured by S6 phosphorylation after stimulation. In contrast, activation of AMPK, an inhibitor of mTORC1 was mostly unaltered in patients compared to controls (Fig. 2).

In conclusion, the present results obtained in septic shock patients show that proximal TCR signaling remains functional in circulating CD4+ T cells from septic shock patients while downstream activation of mTORC1 pathway is markedly diminished. PI3K-Akt pathway integrates signals from both co-activating/inhibitory receptors and an increased expression of such co-inhibitory receptors has been described on circulating $\mathrm{T}$ cells from septic patients [4]. In that respect, this suggests

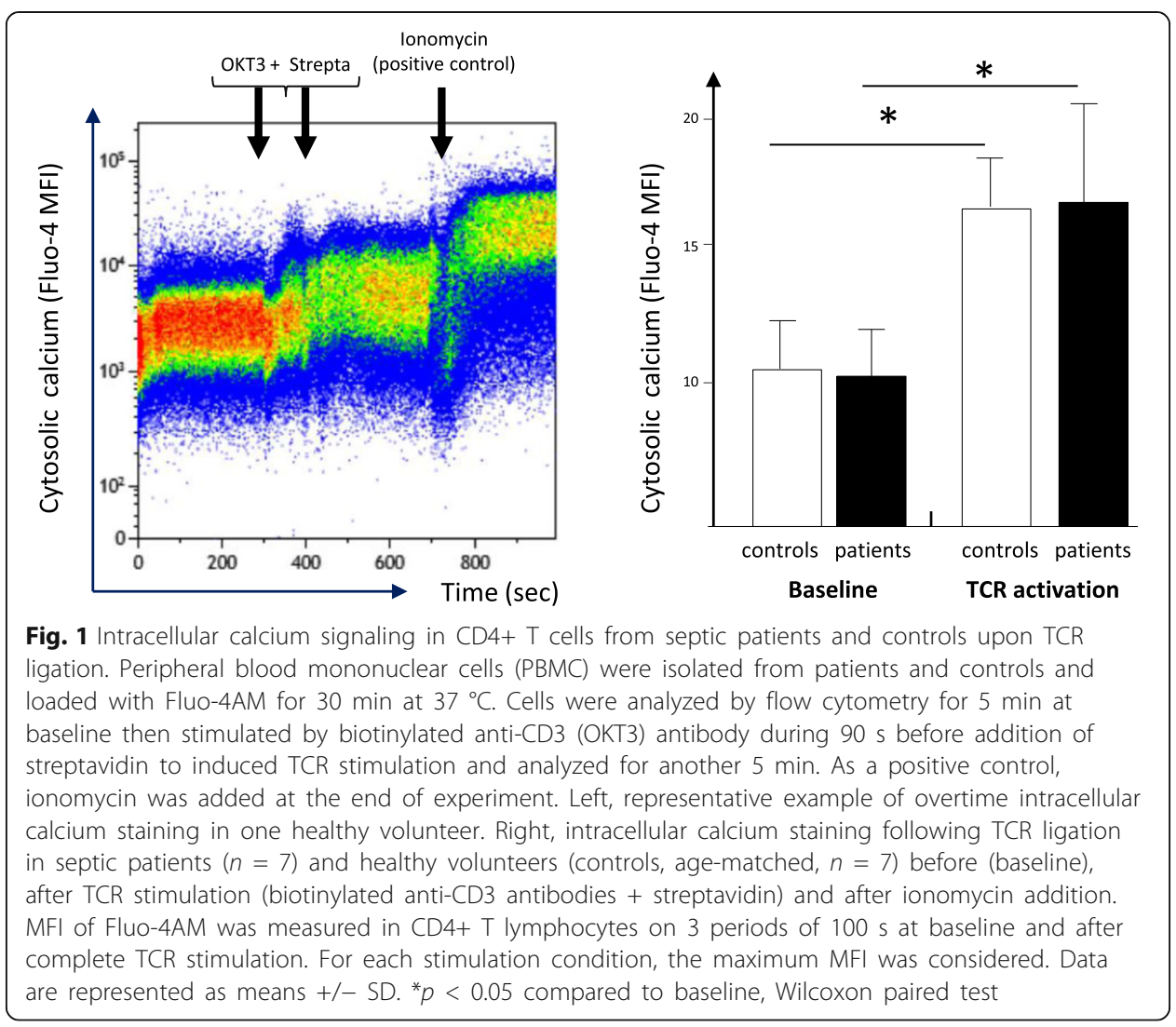




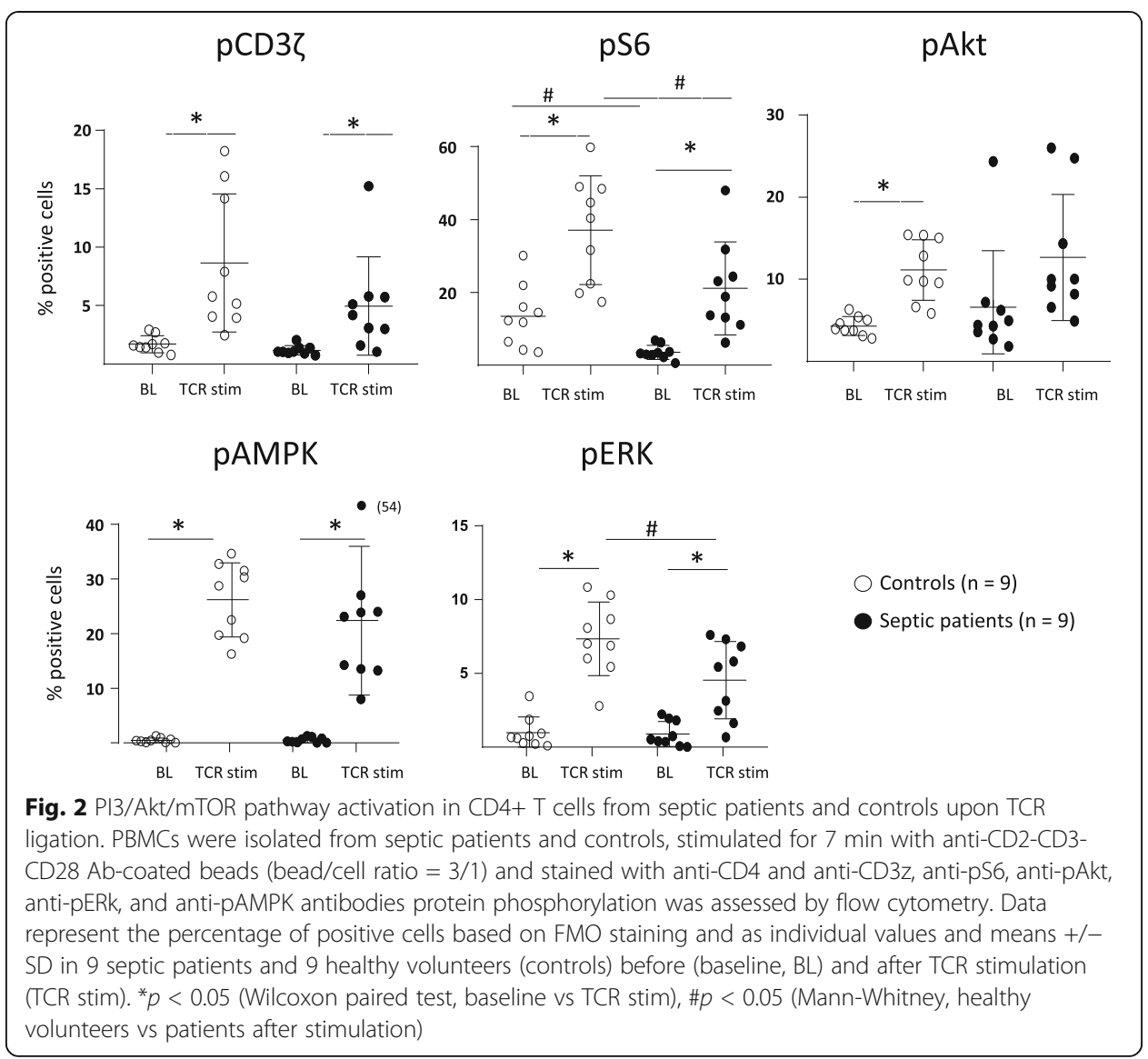

that inhibitory receptors known to block downstream signaling are likely of utmost importance in sepsis-induced T lymphocyte dysfunctions. As TCR from septic lymphocytes remains actionable [5], the present results reinforce the rational for blocking co-inhibitors (e.g., with anti-PD-1) or stimulating mTORC1 (for example with rhIL-7) as reasonable immunoadjuvant approaches to tackle sepsis-induced immunosuppression [6-8].

\section{Supplementary information}

Supplementary information accompanies this paper at https://doi.org/10.1186/s40635-019-0287-5.

Additional file 1: Table S1. Clinical and biological data from septic shock patients.

Abbreviations

anti-PD-1: Anti-Program-Death-1; HLA-DR: Human leukocyte antigen-DR isotype; mTORC1: Mammalian target of rapamycin complex 1; OKT3: Monoclonal antibody anti-CD3 receptor (muromomab); pAkt: Phosphor-Akt (aka phospho-protein kinase B); pAMPK: Phosphor-mitogen-activated protein kinases; pERK: Phosphor-extracellular signalregulated kinases; rhIL-7: Recombinant human interleukin-7; TCR: T cell receptor

Acknowledgements

Not applicable

\section{Authors' contributions}

CDR, KK, MG, EP, FV, and GM designed the experiments. CDR and KK performed the experiments and the statistical analyses. TR, CM, MC, and LA included patients. All authors discussed the data, drafted or revised critically the manuscript for important intellectual content, and approved the final manuscript. 
Funding

This work was supported by Université Lyon 1, Hospices Civils de Lyon, and bioMerieux. bioMérieux had no role in the study design; in the collection, analysis, and interpretation of data; and in writing the manuscript.

\section{Availability of data and materials}

The datasets used and/or analyzed during the current study are available from the corresponding author on reasonable request.

\section{Ethics approval and consent to participate}

Septic shock patients: this project was approved by our Institutional Review Board for ethics ("Comité de Protection des Personnes Sud-Est II", number 11236).This study is registered at the French Ministry of Research and Teaching (\#DC-2008-509), at the Commission Nationale de I'Informatique et des Libertés, and on ClinicalTrials.gov (NCT02803346). Oral information and non-opposition to inclusion in the study were mandatory and recorded in patients' clinical files.

Healthy volunteers: peripheral blood from healthy volunteers was provided by the "Etablissement Français du Sang" from Lyon. According to EFS standardized procedures for blood donation and to provisions of the articles R.1243-49 and following ones of the French public health codes, a written non-opposition to the use of donated blood for research purposes was obtained from HV. The blood donors' personal data were anonymized before being transferred to our research laboratory.

\section{Consent for publication}

Not applicable.

\section{Competing interests}

EP is an employee of bioMérieux. All other authors declare that they have no competing interests.

\section{Author details}

1 EA 7426, Pathophysiology of Injury-Induced Immunosuppression (Université Claude Bernard Lyon 1 - Hospices Civils de Lyon - bioMérieux), Edouard Herriot Hospital, 69437 Lyon, France. ${ }^{2}$ Current address: INSERM U942 MArkers in Stressed COndiTions (MASCOT), Hôpital Lariboisière, Lyon Cedex, 03 Paris, France. ${ }^{3}$ Immunology Laboratory, Hôpital E. Herriot - Hospices Civils de Lyon, 69437 Lyon, France. ${ }^{4}$ Joint Research Unit HCL-bioMérieux, Hôpital Edouard Herriot, 5 place d'Arsonval, 69003 Lyon, France. ${ }^{5}$ Anesthesia and Critical Care Medicine Department, Hospices Civils de Lyon, Edouard Herriot Hospital, 69437 Lyon, France. ${ }^{6}$ Intensive Care Medicine Department, Hospices Civils de Lyon, Edouard Herriot Hospital, 69437 Lyon, France.

Received: 11 October 2019 Accepted: 5 December 2019

Published online: 23 December 2019

\section{References}

1. Venet F, Monneret G (2018) Advances in the understanding and treatment of sepsis-induced immunosuppression. Nat Rev Nephrol 14:121-137

2. Hotchkiss RS, Monneret G, Payen D (2013) Immunosuppression in sepsis: a novel understanding of the disorder and a new therapeutic approach. Lancet Infect Dis 13:260-268

3. Venet F, Demaret J, Blaise BJ, Rouget C, Girardot T, Idealisoa E, Rimmele T, Mallet F, Lepape A, Textoris J, Monneret G (2017) IL-7 restores T lymphocyte immunometabolic failure in septic shock patients through mTOR activation. J Immunol 199:1606-1615

4. Guignant C, Lepape A, Huang X, Kherouf H, Denis L, Poitevin F, Malcus C, Cheron A, Allaouchiche B, Gueyffier F, Ayala A Monneret G, Venet F (2011) Programmed death-1 levels correlate with increased mortality, nosocomial infection and immune dysfunctions in septic shock patients. Crit Care 15:R99

5. Demaret J, Dupont G, Venet F, Friggeri A, Lepape A, Rimmele T, Morel J, Monneret G (2015) STAT5 phosphorylation in T cell subsets from septic patients in response to recombinant human interleukin-7: a pilot study. J Leukoc Biol 97:791-796

6. Francois B, Jeannet R, Daix T, Walton AH, Shotwell MS, Unsinger J, Monneret G, Rimmele T, Blood T, Morre M, Gregoire A, Mayo GA, Blood J, Durum SK, Sherwood ER, Hotchkiss RS (2018) Interleukin-7 restores lymphocytes in septic shock: the IRIS-7 randomized clinical trial. JCI Insight 3:e98960

7. Grimaldi D, Pradier O, Hotchkiss RS, Vincent JL (2017) Nivolumab plus interferon-gamma in the treatment of intractable mucormycosis. Lancet Infect Dis 17:18

8. Hotchkiss RS, Colston E, Yende S, Angus DC, Moldawer LL, Crouser ED, Martin GS, Coopersmith CM, Brakenridge S, Mayr FB, Park PK, Ye J, Catlett IM, Girgis IG, Grasela DM (2019) Immune checkpoint inhibition in sepsis: a phase 1b randomized, placebo-controlled, single ascending dose study of antiprogrammed cell death-ligand 1 antibody (BMS936559). Crit Care Med 47:632-642

\section{Publisher's Note}

